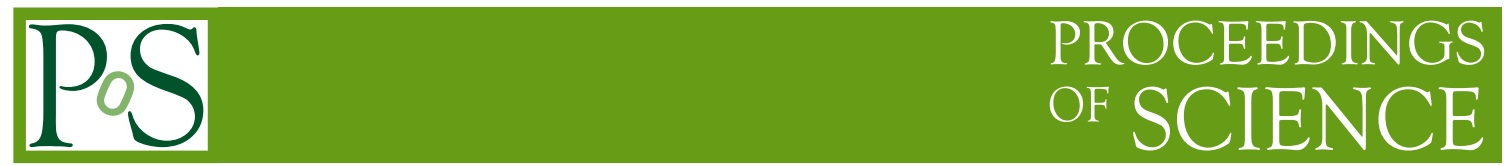

\title{
Composition of the ATLAS Collaboration
}

\section{Joleen Pater* University of Manchester}

E-mail: joleen.pater@manchester.ac.uk

\section{on behalf of the ATLAS Collaboration}

The ATLAS collaboration consists of about 5300 members from 182 institutions in 38 countries. Approximately half of the members of the collaboration are scientific authors of the papers, and there are roughly 1,200 students in the collaboration. This note presents data on the gender and geographical composition of the collaboration; in particular the relative fraction of women is examined at several levels within the organisation of the ATLAS experiment.

38th International Conference on High Energy Physics 3-10 August 2016

Chicago, USA

${ }^{*}$ Speaker. 


\section{Introduction}

The ATLAS Collaboration was founded in 1992 and currently has approximately 5300 members from 94 different nations. In 2015, ATLAS established a Study Group on Diversity[1]. This group's goal is to assess the diversity within the collaboration and make recommendations on how best to support it. This paper will present some of the group's findings on the gender and geographic composition of the collaboration, how people participate, how they are represented in leadership and management roles, and how they are recognised for their contributions.

\section{Composition of the Collaboration}

The ATLAS Study Group on Diversity focused on gender and geography. Available information included a person's Institute of Affiliation, their gender (available choices were male and female), age, profession and nationality. Most of this information was self-declared by the member during the CERN registration process.

Around $80 \%$ of ATLAS members are PhD physicists or physics students; the other $20 \%$ are largely engineers and technicians. There are 2800 scientific authors affiliated with 182 institutions in 38 countries.

About 19\% of the collaboration is female. Table 1 shows the breakdown of the population by profession and gender, from which we see that the fraction of women is larger than average in the physics student and administrative support categories, slightly smaller than average amongst $\mathrm{PhD}$ physicists, and considerably lower than average amongst engineers and technicians.

Although the numbers of people presented in this paper are exact, binomial uncertainties on fractions are shown, to facilitate comparisons with the average and the other categories.

\begin{tabular}{|l|c|c|}
\hline Profession & Number of people (fraction) & Fraction of women (\%) \\
\hline \hline Physicist & $2,237(44 \%)$ & $17 \pm 1$ \\
\hline Physics PhD student & $1,080(21 \%)$ & $24 \pm 1$ \\
Physics master/diploma student & $443(9 \%)$ & $22 \pm 2$ \\
Summer/undergraduate student & $234(5 \%)$ & $27 \pm 3$ \\
Engineering student & $67(1.3 \%)$ & $12 \pm 4$ \\
\hline Engineer & $711(14 \%)$ & $10 \pm 1$ \\
Technician & $210(4 \%)$ & $7 \pm 2$ \\
Administrative support & $78(1.5 \%)$ & $62 \pm 5$ \\
\hline
\end{tabular}

Table 1: Breakdown of the ATLAS Collaboration by profession and gender.

Roughly half of ATLAS members are younger than 35 years of age. As this is a reasonable boundary between people holding short-term positions and those in longer-term positions, this division is used to compare those sub-populations.

Figure 1 shows the correlation between gender and age within ATLAS. The upper histograms use colour to indicate the gender composition; the lower plots show the fraction of women in each bin, with a horizontal blue line depicting the $19 \%$ overall fraction of women within the collaboration. A striking decrease in the fraction of women with age is observed.

Figure 2 shows the geographical diversity of the collaboration; a total of 94 nationalities are represented. The 38 countries shown in blue have at least one ATLAS member institution; the 

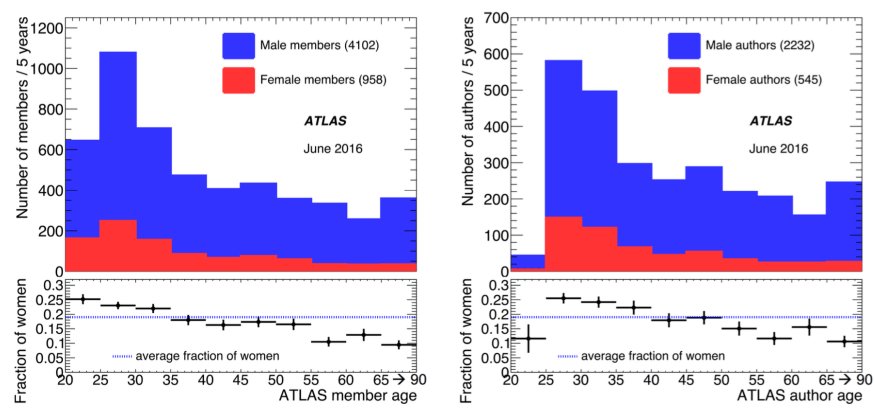

Figure 1: The correlation of gender with age within ATLAS, for all members (left) and for scientific authors only (right).

countries shown in green do not have a member institution, but at least one member with that nationality.

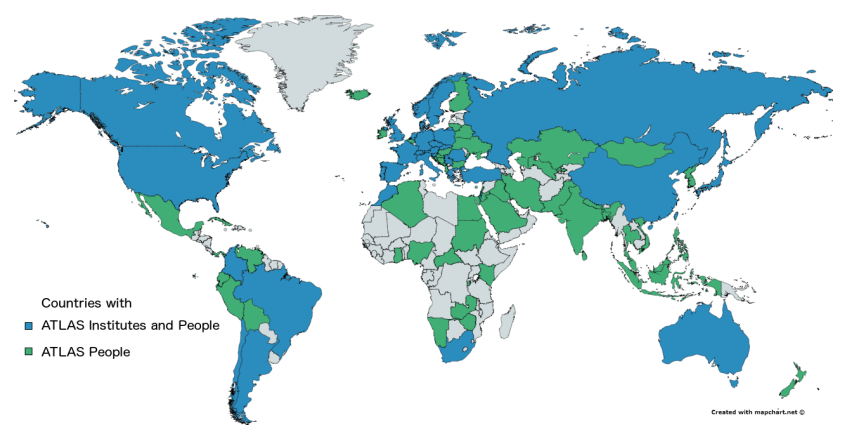

Figure 2: The geographical diversity of the ATLAS Collaboration.

The correlation of gender with region of affiliation was examined by sorting members into six world regions according to their Institute of Affiliation: "Asia" includes Armenia, Azerbaijan, China, Georgia, Japan, and Taiwan; "Eastern Europe" includes Belarus, the Czech Republic, Poland, Romania, Russia (including JINR Dubna), Serbia, Slovakia, and Slovenia; the "Mediterranean" countries are France, Greece, Israel, Italy, Morocco, Portugal, Spain, and Turkey; "North America" is Canada and the USA; "Northern Europe" comprises Austria, Denmark, Germany, the Netherlands, Norway, Sweden, Switzerland (including CERN), and the UK; and the "Southern Hemisphere" countries are Argentina, Australia, Brazil, Chile, Colombia, and South Africa. These regions were defined of course by geographical proximity, but also to create similarly-sized groups with enough statistics to be significant.

As can be seen in Figure 3, the highest fractions of women are in the Mediterranean, Northern European and North American institutions. There are more women in the younger half of the collaboration than the older half, but the regional variation is similar for the two age groups.

\section{Leadership Positions}

The gender composition of leadership positions across the collaboration was examined. Figure 4 shows the relative fraction of men and women in various positions of responsibility. The 

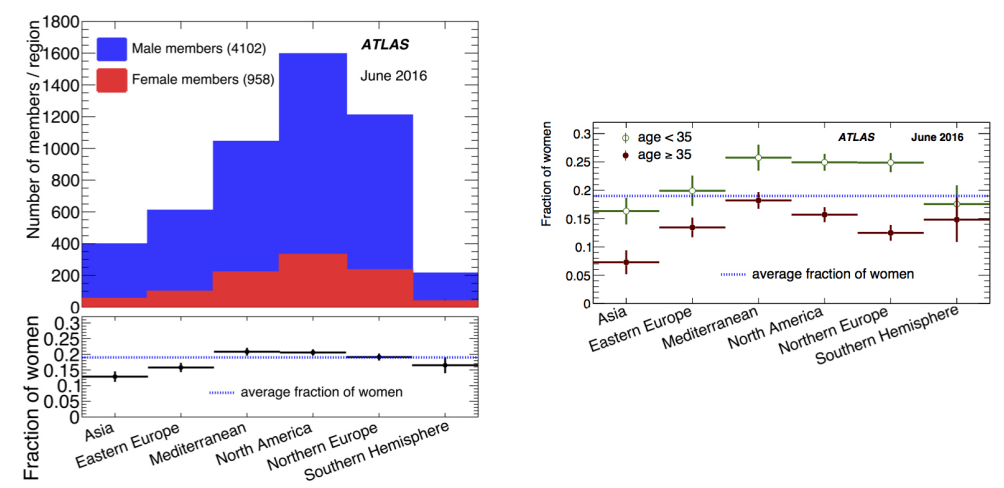

Figure 3: The correlation of gender with region in ATLAS. The left plots show the gender of ATLAS members sorted by the geographical location of their instituties of affiliation. The right plot compares the fraction of women in each region for younger and older members.

bins represent, from left to right: "Top Level Management", which includes the Spokesperson, two deputy Spokespersons, the Technical Coordinator and the Resource Coordinator of the experiment; the "Major Area Coordinators", who are responsible for either a detector or a major activity; the Institution Team Leaders; the Physics Coordination team, comprising the convenors of nine Physics Analysis groups and six Combined Performance groups; numerous leaders in the Trigger, Data Preparation and Computing \& Software areas; subgroup co-convenors within the Physics Analysis and Combined Performance groups; and the Publications Committee (which oversees the process of publishing scientific results in journals) and the Speakers Committee (which is responsible for allocating talks to ATLAS members to show results at conferences and workshops).

As illustrated by Figure 4, women fill leadership roles across the collaboration in proportions consistent with the $19 \%$ overall fraction of women.
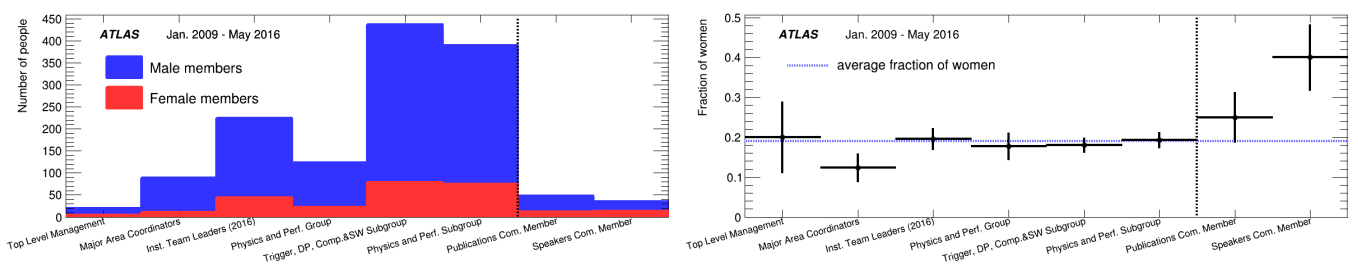

Figure 4: The correlation between leadership and gender in the collaboration.

Figure 5 shows the geographical diversity of the Executive Board (EB), which is the main steering body of the collaboration. The Executive Board is composed of people from the leftmost two bins of Figure 4, plus the chair of the publications committee and three members-at-large from the collaboration. The plot shows the fraction of a region's population that has served on the Executive Board. Two things should be noted here: CERN staff are not included in this plot (they have higher representation at about 5\%), and as of yet there have been no EB members from the Southern Hemisphere institutes, so a 95\% confidence level limit is shown in the corresponding bin. 


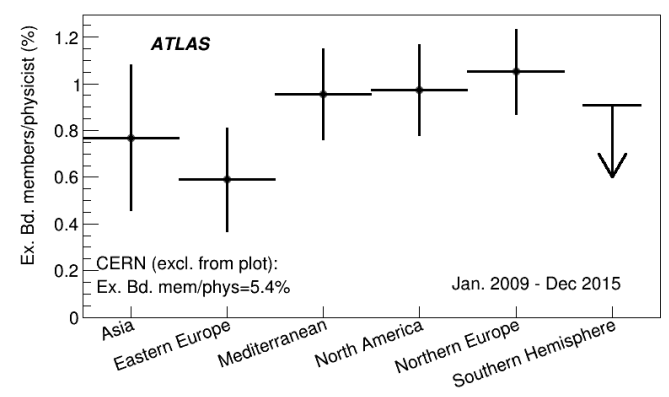

Figure 5: The geographical diversity of the ATLAS Executive Board.

\section{Contributions to the Experiment and Allocation of Talks}

Giving talks at large conferences is an important way in which members' contributions are recognised. In ATLAS, talks are allocated by a Speakers Committee using a consistent procedure that has been designed to be as equitable as possible. Activity Coordinators, Project Leaders and Institute Team Leaders nominate authors who are associated with their project or institute. The total number of nominations a person receives is important, as is the priority of the nomination, which can range from 1 (highest) to 5 (lowest). Figure 6 shows the number of nominations received by authors in ATLAS, separated by gender, and also the priority of the nominations given by the Physics Coordination team. For reasons which are not clear, women receive more nominations than men, and they also tend to receive higher-priority nominations.

The amount of time a person contributes to operational tasks (such as shifts, data quality checks, etc.) is also considered in awarding talks. The rightmost plot of Figure 6 shows that men and women contribute proportionately to operations.
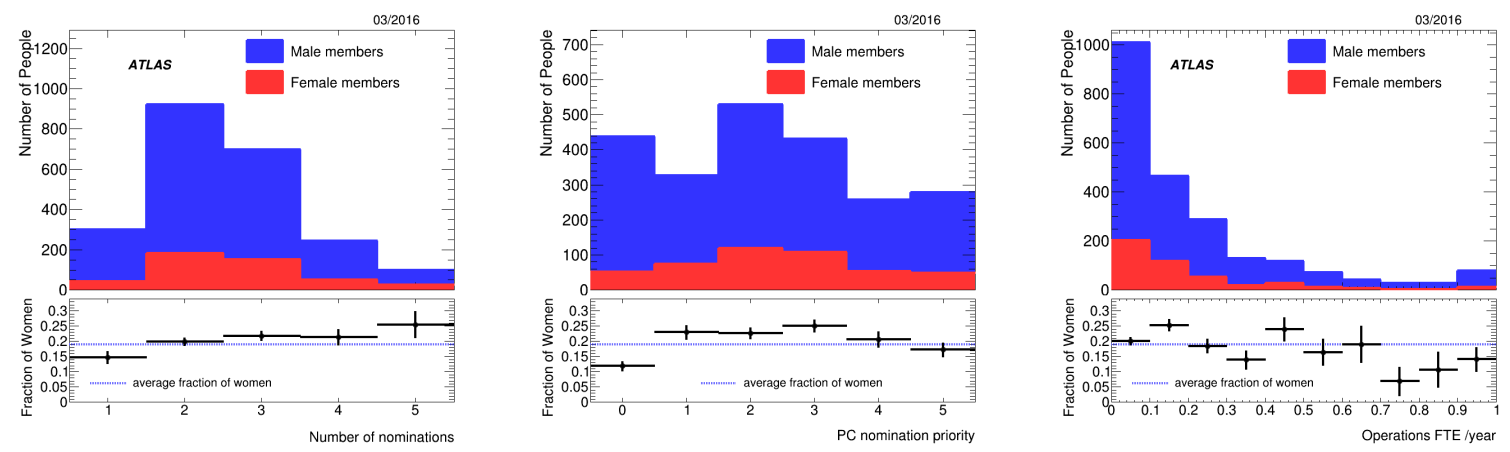

Figure 6: Some factors which influence the awarding of talks to ATLAS authors. From left to right: the number of talk nominations received, the priority of the nominations from the Physics Coordinator, and the amount of time spent on operational tasks.

Table 2 shows the results of talk allocation in ATLAS since 2010, presented according to the speaker's gender and time as an ATLAS member. Women have given proportionately more talks than men, at least among longer-time collaborators (for students it is more consistent). This makes sense when put together with the information in the previous paragraph - we know that this is at least partly due to the fact that women receive more and generally stronger nominations for talks. 
The regional affiliation of talk allocation has also been examined; no significant correlation was seen.

\begin{tabular}{|l|c|c|c|}
\hline Selection & time as ATLAS author & Fraction of men (\%) & Fraction of women (\%) \\
\hline \hline Physicist & 2-7 years & $83 \pm 3$ & $91 \pm 4$ \\
Physicist & $7-15$ years & $81 \pm 2$ & $94 \pm 3$ \\
Physicist & $>15$ years & $55 \pm 4$ & $77 \pm 8$ \\
Student & $>2$ years & $62 \pm 5$ & $57 \pm 8$ \\
\hline
\end{tabular}

Table 2: The fraction of men and women who have given ATLAS talks in the last six years, loosely as a function of how long they've been on ATLAS.

ATLAS holds week-long collaboration meetings three times per year. At these meetings, plenary talks are given by members who are playing key roles and so this represents another form of recognition of outstanding work. Figure 7 shows the time-wise evolution of ATLAS-week speakers, separated by gender. The fraction of female speakers has increased with time, and while older data on gender composition is not available for comparison with this plot, in recent years the fraction of talks given by women is consistent with the current total fraction of women in the collaboration.
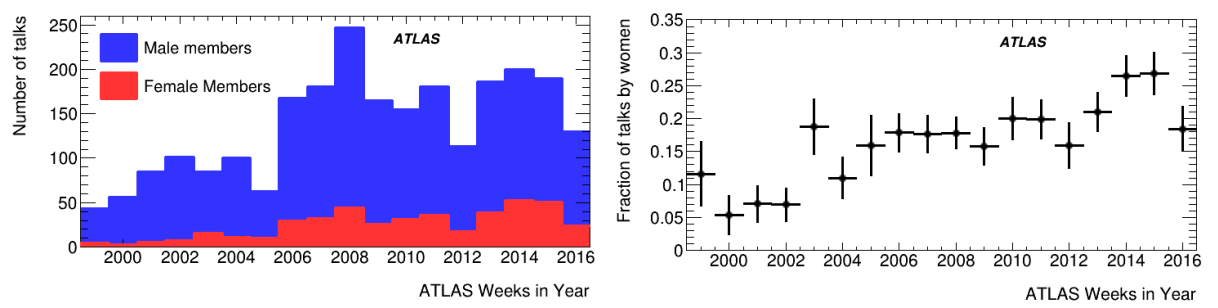

Figure 7: The time evolution of plenary speakers at ATLAS weeks, separated by gender. Left: the overall number of talks; right: the fraction of female speakers.

\section{Summary and Conclusions}

This paper presented data on the gender and geographical composition of the ATLAS Collaboration. ATLAS currently has about 5300 members, 19\% of whom are female. Women contribute proportionately to the experiment and are represented proportionately in leadership roles and as speakers at international conferences and in internal meetings. There are 94 nationalities represented on ATLAS, and the various world regions are also represented proportionately in leadership positions and recognition.

A decrease in the fraction of women with age is observed. The cause of this cannot be determined with the information available; it may simply be due to an increase over time in women's participation in physics and engineering. It could also be due to the "leaky pipeline effect" (the continuous loss of women as they climb the career ladder), or a combination of both these effects.

There are other aspects of diversity within ATLAS which are not addressed in this paper, largely due to limitations in the available data.

\section{References}

[1] The ATLAS Collaboration, CERN, Geneva, 2016, ATL-GEN-PUB-2016-001. 